\title{
Fokker-Planck analysis of stochastic coherence in models of an excitable neuron with noise in both fast and slow dynamics
}

\author{
Robert C. Hilborn ${ }^{1, *}$ and Rebecca J. Erwin ${ }^{2}$ \\ ${ }^{1}$ Department of Physics, Amherst College, Amherst, Massachusetts 01002, USA \\ ${ }^{2}$ Department of Physics, California Institute of Technology, Pasadena, California 91125, USA \\ (Received 25 May 2005; revised manuscript received 18 July 2005; published 29 September 2005)
}

\begin{abstract}
We provide a detailed and quantitative Fokker-Planck analysis of noise-induced periodicity (stochastic coherence, also known as coherence resonance) in both a discrete-time model and a continuous-time model of excitable neurons. In particular, we show that one-dimensional models can explain why the effects of noise added to the fast and slow dynamics of the models are dramatically different. We argue that such effects should occur in any excitable system with two or more distinct time scales and need to be taken into account in experiments investigating stochastic coherence.
\end{abstract}

DOI: 10.1103/PhysRevE.72.031112

PACS number(s): 05.40.- a, 05.45.-a, 87.17.Nn

\section{INTRODUCTION}

Noise can often play a constructive role in nonlinear dynamical systems. Under appropriate conditions, noise can enhance the response of a nonlinear system to an external signal, an effect known as stochastic resonance [1]. For other systems, noise can enhance the periodicity of an oscillatory component of the system's behavior, an effect known as autonomous stochastic resonance [2,3], coherence resonance [4], or stochastic coherence [5]. We prefer, and will use, the term "stochastic coherence" instead of "coherence resonance" because the former emphasizes the stochastic inducement of periodicity ("coherence"). There is no explicit "resonance" effect.

Stochastic coherence has been demonstrated in models of single excitable systems $[3,4,6-12]$, coupled excitable systems [13-16], coupled chaotic systems $[17,18]$, spatiotemporal arrays [19-21], systems with time delays [22], and a few experimental systems [14,23-29]. Reference [30] provides a review of stochastic coherence (and stochastic resonance) in excitable systems.

In a recent paper [31], we demonstrated an additional feature of stochastic coherence in excitable systems with two distinct time scales (a fast scale and a slow scale): adding noise to the dynamics of the fast variable produces a stochastic coherence effect significantly different from that produced by adding noise to the dynamics of the slow variable. We demonstrated the effect in both a discrete-time model [32] and a continuous-time model [33] of excitable neurons. This fast vs slow effect enriches the repertoire of noise-induced phenomena. The effect should also be important in the interpretation of experimental observations of stochastic coherence in any system with multiple time scales.

In this paper, we demonstrate this fast vs slow noise effect over a wider range of parameter values and also with a different continuous-time model. We also present a detailed Fokker-Planck analysis that allows for noise in both the fast and slow dynamics and provides a quantitative description of

\footnotetext{
*Electronic address: rchilborn@amherst.edu
}

the fast and slow noise effects in both the discrete-time and continuous-time models.

In Sec. II, we introduce the discrete-time neuron model. Section III displays the enhanced periodicity of the discretetime dynamics of that model as a function of noise amplitude. In Sec. IV, we provide a quantitative explanation of these effects for the discrete-time model using a onedimensional Fokker-Planck first-exit-time formulation. Section $\mathrm{V}$ introduces the FitzHugh-Nagumo model, a continuous-time neuron model, and we discuss stochastic coherence in its behavior as a function of noise amplitude. A Fokker-Planck analysis of the FitzHugh-Nagumo model's stochastic coherence is given in Sec. VI. Section VII concludes the paper with some discussion of the results and possible applications to experimental systems. We also consider the limitations of the one-dimensional models. In a subsequent paper [34], we will expand the analysis to general systems with two distinct time scales.

\section{RULKOV MODEL}

Real neurons often display (at least) two time scales [35]: fast dynamics corresponding to action potentials and slow dynamics corresponding to gating-ion concentration variations. We first consider a discrete-time (iterated map) neuron model, recently introduced by Rulkov [32], that captures this fast-slow distinction. The behavior of this model, though not directly linked to the physiology of neurons, nevertheless mimics that of actual neurons [36,37]. The model has two dynamical variables: $x_{n}$, corresponding to the membrane voltage in a neuron, and $y_{n}$, corresponding to a gating-ion concentration (usually $\mathrm{Ca}^{2+}$ in actual neurons). With additive noise terms, the model takes the following form:

$$
\begin{gathered}
x_{n+1}=f\left(x_{n}, y_{n}\right)+\sqrt{D_{x}} \xi_{x n}=\frac{\alpha}{1+x_{n}^{2}}+y_{n}+\sqrt{D_{x}} \xi_{x n}, \\
y_{n+1}=g\left(x_{n}, y_{n}\right)+\sqrt{D_{y}} \xi_{y n}=y_{n}-\beta x_{n}-\sigma+\sqrt{D_{y}} \xi_{y n},
\end{gathered}
$$

where $\alpha, \beta$, and $\sigma$ are parameters that set the model's deterministic behavior through the functions $f$ and $g . n$ is the 


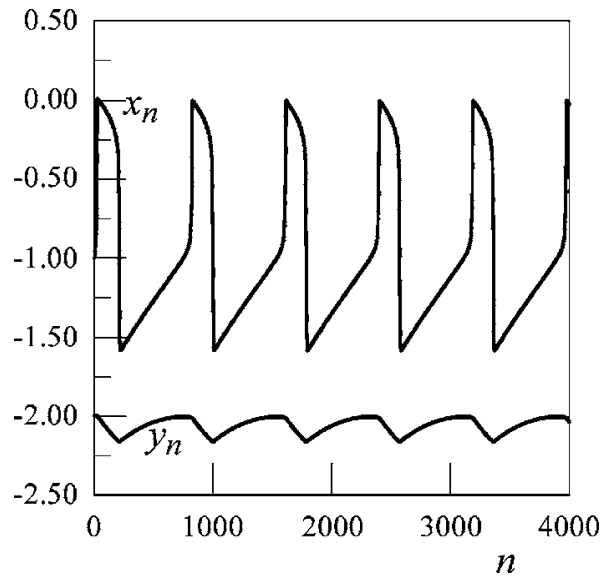

FIG. 1. A plot of $x_{n}$ and $y_{n}$ as a function of iteration number $n$ for the Rulkov model with $\alpha=2.02$ and $\beta=\sigma=10^{-3}$. The noise terms have been set to zero.

iteration number (discrete-time index). The two independent noise terms $\xi_{x n}$ and $\xi_{y n}$ have zero mean and variance $D$. The corresponding probability distributions are taken to be Gaussian. Reference [38] contains an elementary introduction to the dynamics of the model.

In spite of its simplicity, the Rulkov model captures many of the important features of neuron behavior. With adjustment of the parameter $\alpha$, the model can have excitable behavior $(\alpha<2)$, sustained periodic pulses $(2<\alpha<4)$, chaotic bursts of pulses $(4<\alpha<4.6)$, sustained chaotic pulses (4.6 $<\alpha<7)$, and complicated sequences of periodic pulses $(\alpha$ $>7$ ). (For $2.6<\alpha<4$, the periodic pulses have a complex substructure.) Figure 1 illustrates the behavior of the system for $\alpha=2.02$, which puts the model in a periodic pulse regime. We see that $x$ mimics the fast rise and fall of action potentials while $y$ has more limited and smoother excursions, characteristic of gating-ion concentration variations near nerve cell membranes. Since the parameters $\beta$ and $\sigma$ are small, the variations in $y$ are slower than those for $x$.

For $\alpha<1.998$, the behavior consists of damped oscillations as the system approaches the (no-noise) fixed point at $x=-\sigma / \beta=-1$ and $y=-1-\alpha / 2$. This behavior is illustrated in Fig. 2. The fixed point is a spiral attractor. For $1.998<\alpha$ $<1.99900977$, the behavior consists of sustained, smallamplitude oscillations around the fixed point. These oscillations are analogous to the so-called subthreshold oscillations observed in real neurons [39,40]. In this paper we focus on the excitable regime with $\alpha<1.998$.

\section{STOCHASTIC COHERENCE IN THE RULKOV MODEL}

We now turn to the behavior of the Rulkov model, Eqs. (1) and (2), with nonzero noise terms and explore the phenomenology of stochastic coherence in this model. We start with $\alpha=1.99$, which puts the system in an excitable regime. If no noise is present, the long-term behavior is just a steady state at the fixed point values $x^{*}=-\sigma / \beta=-1$ and $y^{*}=-1$ $-\alpha / 2$. With noise present, however, a large noise "kick" can move the system sufficiently far from the no-noise fixed
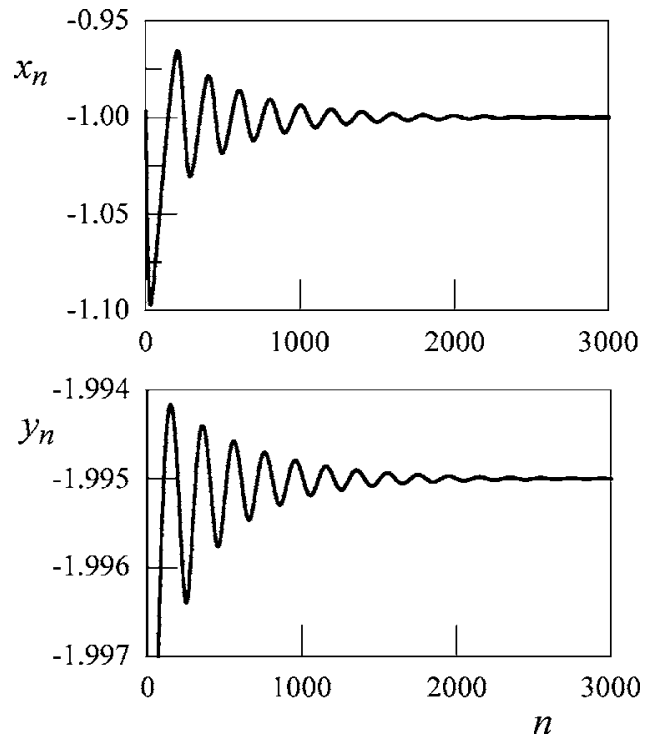

FIG. 2. Rulkov model trajectories $x_{n}$ and $y_{n}$ as a function of iteration number $n$ for $\alpha=1.99$ and $\beta=\sigma=10^{-3}$. The trajectories approach the fixed point, whose coordinates are $x^{*}=-1$ and $y^{*}=$ $-1-\alpha / 2$. The noise terms have been set to zero.

point, leading to a large excursion through state space (a pulse) before the system returns to the fixed point. Figure 3 illustrates noise-induced pulses for $D_{x}=1.6 \times 10^{-5}$ and $D_{y}$ $=0$. The number of pulses per unit time is described approximately by a Poisson distribution.

As the noise level increases, the pulses first become more regular as illustrated in Fig. 4. For still larger noise amplitudes, the regularity of the pulses decreases.

We now turn to stochastic coherence, the main topic of this paper. Stochastic coherence (also known as coherence resonance [4]) is an enhanced periodicity of a system's behavior with increasing noise intensity. For an excitable sys-

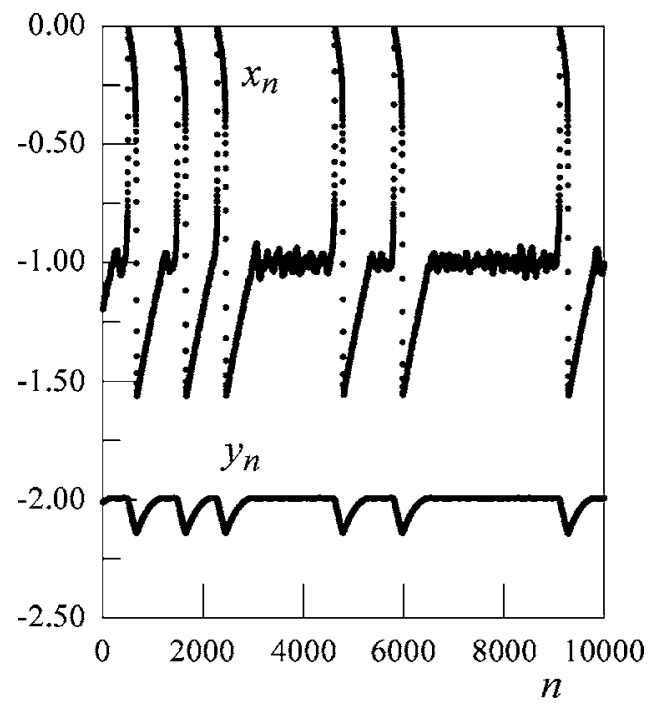

FIG. 3. Noise-induced pulses in the Rulkov model for $\alpha$ $=1.99, \beta=\sigma=10^{-3}, D_{x}=1.6 \times 10^{-5}$, and $D_{y}=0$, after transients have died out. The upper trace is $x_{n}$ and the lower trace is $y_{n}$ as a function of iteration number $n$. 


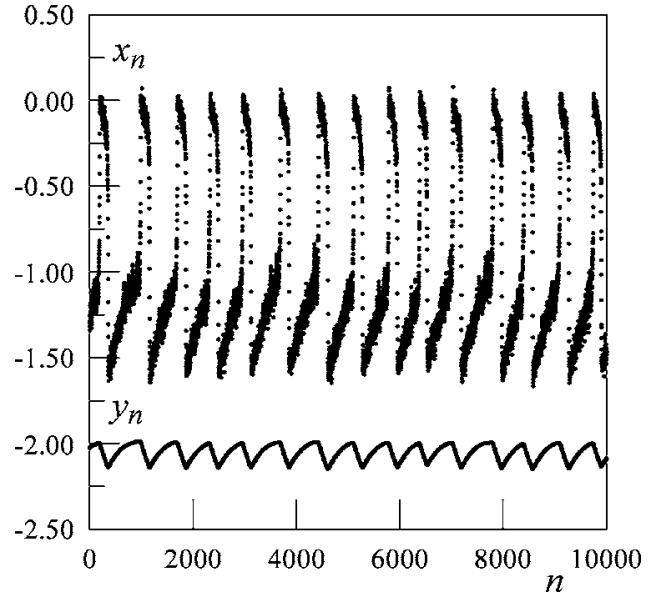

FIG. 4. Noise-induced pulses in the Rulkov model for $\alpha$ $=1.99, \beta=\sigma=10^{-3}, D_{x}=9 \times 10^{-4}$, and $D_{y}=0$.

tem, we quantify the periodicity by finding the ratio of the mean time between noise-induced pulses $\langle T\rangle$ to the variance of that time $\operatorname{var}(T)$. The "regularity" $R[41]$ is defined as

$$
R=\frac{\langle T\rangle}{\sqrt{\operatorname{var}(T)}}
$$

Note that $R$ is the reciprocal of the quantity used by Pikovsky and Kurths [4] to characterize coherence resonance. The reciprocal is the same as the "coefficient of variation" often employed in neuroscience [42] as a measure of interspike interval regularity. Stochastic coherence may occur in an excitable system because the time between noise-induced pulses decreases as the noise intensity increases, while, for small noise intensities, the excursion time is little affected by noise. This effect tends to increase the regularity of the system's behavior. For larger noise intensities, the system is "activated" as soon as the excursion phase ends, and the noise starts to cause significant fluctuations in the excursion time itself, thus tending to decrease the regularity. The combination of these two trends leads to a maximum in the regularity as a function of noise intensity.

Figure 5 shows the regularity for the Rulkov model as a function of noise intensity for two cases: (1) noise added only to the dynamics of $y$ (the slow variable) and (2) noise added only to the dynamics of $x$ (the fast variable). Each data point represents the average over ten noise realizations, each of which consists of $5 \times 10^{5}$ iterations of Eqs. (1) and (2) after allowing transients to die out. We note two important features. First, when noise is added only to the slow variable, the relevant range of noise intensities is about three orders of magnitude smaller than for the case of noise added only to the fast variable. Second, the maximum size of the regularity is significantly smaller for the first case (noise added only to $y$ ) than for the second (noise added only to $x$ ). Three other trends should also be noted. (1) For smaller values of $\alpha$, the system's fixed point is further from the self-sustained pulsing mode and larger noise amplitudes are required to achieve the maximum in the regularity. (2) The maximum value of the regularity decreases as $\alpha$ decreases. (3) For smaller values of

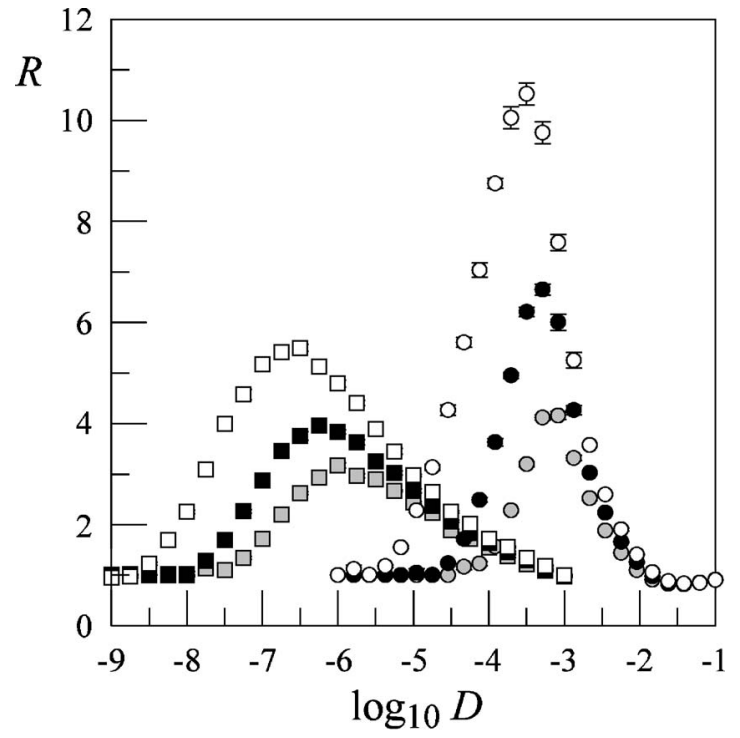

FIG. 5. The regularity $R$ for the Rulkov model, Eqs. (1) and (2), plotted as a function of the logarithm of the noise variance. The squares (on the left) are data for $D_{x}=0$. The circles (on the right) are data for $D_{y}=0$. Open symbols: $\alpha=1.99$. Black symbols: $\alpha=1.95$. Gray symbols: $\alpha=1.91$. Here, we use $\beta=\sigma=10^{-3}$. The uncertainty bars indicate the standard deviation of the mean over ten noise realizations and are, in most cases, smaller than the plotted symbols.

$\alpha$, the difference in the maximum regularity for $x$ noise as compared to that for $y$ noise decreases. In Sec. IV we will provide a quantitative explanation of these effects.

Figure 6 illustrates the regularity of the Rulkov model with $\alpha=1.99$ when both noise sources are present. Two trends should be noted. First, considering the regularity as a function of $D_{x}$ for fixed values of $D_{y}$, we see that the maximum value of $R$ drops rapidly as $D_{y}$ increases. In particular, for $D_{y}>10^{-6}, R$ monotonically decreases as $D_{x}$ increases. Second, consider $R$ as a function of $D_{y}$ for various values of $D_{x}$. As $D_{x}$ increases, the location of the maximum value of $R$ shifts to smaller $D_{y}$ values. For $D_{x}>10^{-3}, R$ decreases monotonically for increasing $D_{y}$.

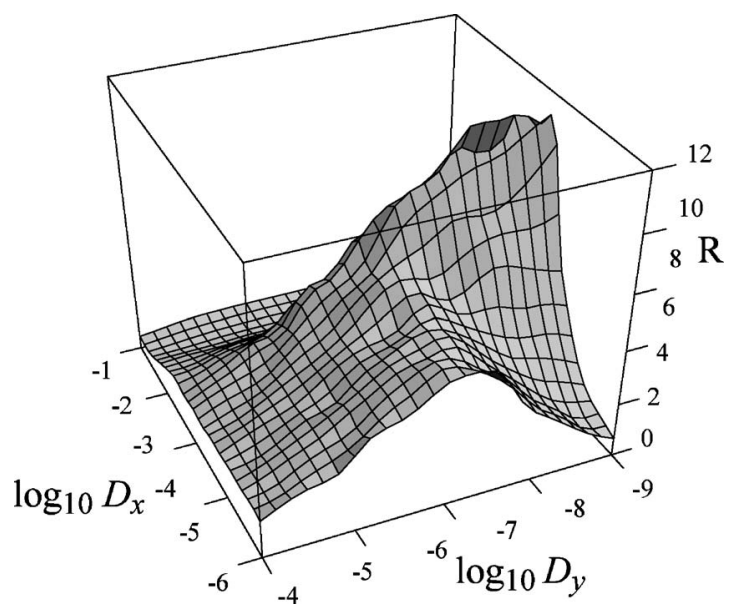

FIG. 6. The regularity $R$ for the Rulkov model, Eqs. (1) and (2), as a function of $\log _{10} D_{x}$ and $\log _{10} D_{y}$ with $\alpha=1.99, \beta=\sigma=0.001$. 


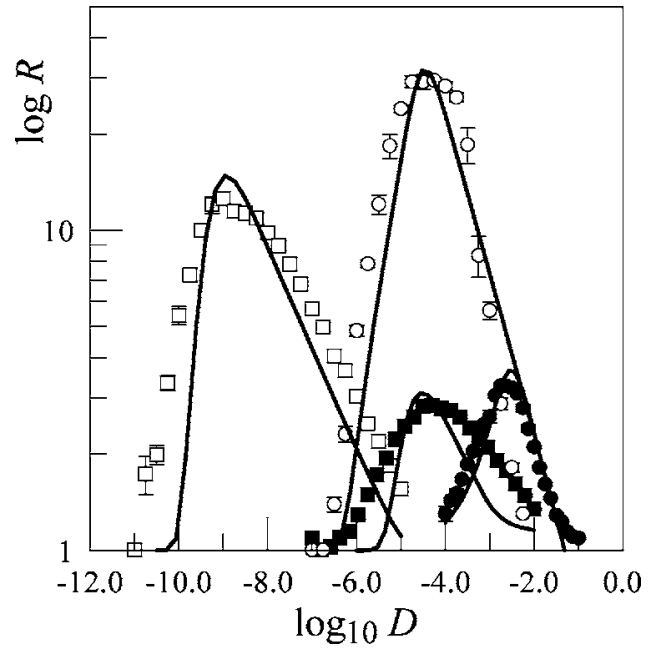

FIG. 7. The logarithm of the regularity $R$ plotted as a function of $\log _{10} D$ for $\beta=\sigma=10^{-4}$ (open symbols) and $\beta=\sigma=10^{-2}$ (solid symbols) for $\alpha=1.99$. For each data set, on the left (squares), noise is added to $y$ alone. On the right (circles) noise is added to $x$ alone. The uncertainty bars indicate the standard deviation of the mean over ten noise realizations. The solid curves are the results of the Fokker-Planck analysis described in Sec. IV.

As we mentioned previously, $\beta$ and $\sigma$ control the timescale separation between the fast and slow dynamics. If $\beta$ and $\sigma$ become smaller, the time-scale separation increases. Figure 7 displays the regularity $R$ as a function of noise amplitude for $\beta=\sigma=10^{-4}$ and $\beta=\sigma=10^{-2}$. (Note the logarithmic scale for $R$.) We see that for smaller values of $\beta$ and $\sigma$, the difference in response to noise in the fast dynamics versus noise in the slow dynamics is enhanced: the ratio of the two maxima is larger and the noise scales are further separated. For $\beta=\sigma=10^{-2}$, the difference between fast and slow noise effects is diminished. In the next section we turn to a theoretical explanation of these effects.

\section{FOKKER-PLANCK ANALYSIS OF STOCHASTIC COHERENCE FOR THE RULKOV MODEL}

\section{A. General comments}

To provide a deeper and more quantitative understanding of the noise-induced periodicity (stochastic coherence), we make use of a Fokker-Planck analysis of the dynamics [43-46]. The general strategy of a Fokker-Planck analysis is to follow the time evolution of a distribution of system points under the action of both the deterministic part of the evolution and the stochastic part. From the evolution of this probability distribution, we can determine the average time behavior of the system and hence the coherence or, more precisely, the regularity of the noise-induced pulses. Our approach is similar in spirit to that used previously by other authors $[4,9,47]$ for the FitzHugh-Nagumo neuron model. Those authors, however, considered the case in which noise appears only in the dynamics of the slow variable. In this paper, we extend the analysis to a discrete-time model and we allow for noise in the dynamics of both the fast and slow variables.
To understand the dynamics, we divide the Rulkov model behavior into three temporal phases and ignore the relatively fast transitions between the phases. (Reference [9] gives a detailed justification of this approach.) The "activation phase" occurs near the no-noise fixed point at $x^{*}=-1$ and $y^{*}=-1-\alpha / 2$. The excursion itself is divided into two parts: a "pulse phase" with $x \approx 0$ and a "recovery phase" during which $x$ increases from about -1.5 to -1 (the location of the no-noise fixed point) as seen in Fig. 3. The system then resides in the neighborhood of the no-noise fixed point until a sufficiently large noise fluctuation allows escape from the "basin of attraction" of the fixed point. We note that the basin of attraction for the fixed point is actually the entire state space, but we distinguish between initial conditions that lead to trajectories that stay in the neighborhood of the fixed point and those that lead to a long excursion through state space before returning to the fixed point.

For the Rulkov model, we should, in principle, use a twodimensional, discrete-time Fokker-Planck analysis with a distribution function for the two dynamical variables $x$ and $y$. Here we will use continuous-time models because the relative change in variables from one iteration to the next is small. Methods for handling multidimensional FokkerPlanck analyses and the corresponding first-exit-time problem are given in standard references dealing with stochastic processes [43-46]. If the time scales between the fast variable and the slow variable are sufficiently distinct, however, we can use the method of "adiabatic elimination" to remove the fast variable from the analysis: we assume that the fast variable quickly relaxes to the "equilibrium" value associated with the present value of the slow variable. In that case, the problem reduces to a one-dimensional model. Below we shall show that we can also use a one-dimensional model when noise is added only to the fast dynamics. This procedure, as we shall demonstrate, gives an adequate, but not perfect, account of stochastic coherence in the Rulkov and FitzHugh-Nagumo models. The limitations of this approach will be discussed in Sec. VII.

\section{B. Fast and slow decomposition}

First, we consider a general two-dimensional dynamical system with a fast variable $x$ and a slow variable $y$. The multi-time-scale dynamics can be written in the following form:

$$
\begin{gathered}
\frac{d x}{d t}=f_{x}(x, y), \\
\frac{d y}{d t}=\varepsilon f_{y}(x, y),
\end{gathered}
$$

where $\varepsilon \ll 1$ sets the time-scale separation. The equation for the time derivative of $y$ is said to form a "singular perturbation" for the $x$ dynamics, because in the limit $\varepsilon \rightarrow 0$, the two-dimensional system turns into a one-dimensional system. We can approach the dynamics in two ways. If we set $\varepsilon=0$, then we have the one-dimensional system 


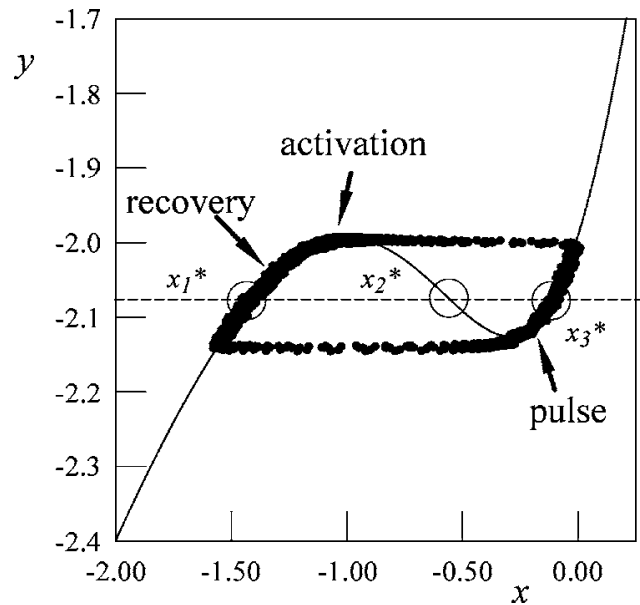

FIG. 8. Rulkov model state-space trajectories (heavy symbols) for noise-induced pulses with $D_{x}=10^{-4}$ and $D_{y}=0$. The $x$ nullcline is indicated by the thin curve. Here we use $\alpha=1.99, \beta=\sigma=0.001$. The state-space locations of the activation, pulse, and recovery phases are indicated. The larger circles indicate the three fixed points of Eq. (1) with $y$ viewed as a fixed parameter.

$$
\frac{d x}{d t}=f_{x}(x, y)
$$

with the constraint $\dot{y}=0$; that is, we treat $y$ as a fixed parameter. On the other hand with $\varepsilon$ small but finite, we observe that $x$ relaxes quickly to a value that satisfies $f_{x}(x, y)=0$, which gives a state-space curve traditionally called the $x$ nullcline. (The curve is sometimes called the "slow manifold.") As $y$ evolves, $x$ tracks along the $x$ nullcline with occasional jumps between different branches of the nullcline. A specific example will be given below.

The slow manifold idea can be approached from another direction by changing the time variable to $\tau=\varepsilon t$ in which case Eq. (4) becomes

$$
\begin{gathered}
\varepsilon \frac{d x}{d \tau}=f_{x}(x, y), \\
\frac{d y}{d \tau}=f_{y}(x, y) .
\end{gathered}
$$

The new "slowed" time scale allows us to focus on the $y$ dynamics. Setting $\varepsilon=0$, we end up with a one-dimensional system for $y$ with the constraint $f_{x}(x, y)=0$, as before. We will use these ideas below to separate the dynamics for the Rulkov model (and later for the FitzHugh-Nagumo model) into one-dimensional subsystems.

An examination of the state-space trajectories for the system indicates that a one-dimensional approximation is valid in the parameter range considered in this paper because of the difference in time scales for the fast variable $(x)$ and the slow variable $(y)$. Figure 8 shows state-space trajectories for the noise-induced pulses for the Rulkov model. The trajectories closely track the $x$ nullcline except for rapid jumps between the branches.

\section{First-exit times}

As we mentioned previously, for small deviations from the no-noise fixed point in the excitable regime, the Rulkov model exhibits small-amplitude damped oscillations relaxing to the no-noise fixed point. Here we will ignore those oscillations. If the oscillation period is comparable to the pulse length, an "anticoherence resonance" effect can occur [47]: decreasing noise can lead to a regularity $R<1$. That is, the regularity can be less than that for a Poisson distribution of spikes. Our data do not exhibit such an effect because, for the parameter values chosen, the oscillation period is shorter than the pulse length.

The crucial ideas for the Fokker-Planck analysis are to divide the behavior into distinct temporal phases $[4,9,47]$, to model the dynamics in each of the phases (in our case an activation phase, a pulse phase, and a recovery phase), and to solve the corresponding first-exit-time problems for the mean and variance of the time spent in each phase. The measure of the overall regularity of the system's behavior is given by

$$
R=\frac{\left\langle T_{a}\right\rangle+\left\langle T_{r}\right\rangle+\left\langle T_{p}\right\rangle}{\sqrt{\operatorname{var}\left(T_{a}\right)+\operatorname{var}\left(T_{r}\right)+\operatorname{var}\left(T_{p}\right)}},
$$

where $\left\langle T_{a}\right\rangle$ is the mean activation time and $\operatorname{var}\left(T_{a}\right)$ its variance, with analogous definitions for the pulse and recovery phases. In writing Eq. (7), we are assuming that the variances add, an appropriate assumption for either Gaussian or exponential distributions.

When noise is added to only one of the variables, we model the dynamics of each of the phases with a Langevin equation of the form

$$
\dot{z}=-\frac{d U(z)}{d z}+\sqrt{D} \xi(t),
$$

where $z$ can be either the $x$ or $y$ variable. $U(z)$ is a potential function that describes the deterministic part of the time evolution, while $\xi(t)$ describes the stochastic evolution. We assume that the noise term is a white-noise, Gaussiandistributed variable with a mean of zero and a variance given by $D$. (Empirically, we have found that the simulation results are essentially the same if we use bounded white noise, colored noise with a correlation time short compared to the pulse length, or "noise" produced by deterministic chaotic dynamics. In this paper we restrict ourselves to white, Gaussian-distributed noise.) Once we have the potential in hand, we can calculate the mean first-passage time $\langle T\rangle$ and its second moment $\left\langle T^{2}\right\rangle$ from the standard expressions for a one-dimensional first-exit-time problem [43-46] in the Fokker-Planck approximation:

$$
\begin{gathered}
\langle T(w)\rangle=\frac{2}{D} \int_{w}^{a} d u e^{2 U(u) / D} \int_{b}^{u} d v e^{-2 U(v) / D} \\
\left\langle T^{2}(w)\right\rangle=\frac{4}{D} \int_{w}^{a} d u e^{2 U(u) / D} \int_{b}^{u} d v e^{-2 U(v) / D} T(v) .
\end{gathered}
$$

Here $a$ is the absorbing boundary for the region, $b$ is the reflecting boundary, and $w$ is the injection point (initial loca- 
tion). In writing Eqs. (9) and (10), we have assumed that $b$ $\leqslant w<a$. For $a<w \leqslant b$, the limits of integration need to be adjusted accordingly [43]. The average is taken with respect to the noise realizations. We note that if $U$ is a double-well potential, Eqs. (9) and (10) reduce to the Kramers form [43] for small noise amplitudes.

\section{Analysis for $D_{x}=0$}

To analyze stochastic coherence in the Rulkov model, we first treat the case in which noise is present only in the dynamics of the slow variable $y$ in Eqs. (1) and (2). This is the case treated by Ref. [9] but with a different (but equivalent) formulation of the potential function. We assume that there is a sufficient separation of time scales so that $y$ in Eq. (1) can be treated as a slowly varying parameter and that the behavior of $x$ is "slaved" [48] to that of $y$. For the discrete-time Rulkov system given by Eqs. (1) and (2), the nullclines are the curves in state space that satisfy

$$
x_{n+1}-x_{n}=\frac{\alpha}{1+x_{n}^{2}}+y_{n}-x_{n}=0
$$

and

$$
y_{n+1}-y_{n}=-\beta x_{n}-\sigma=0 .
$$

The $y$ nullcline Eq. (12) is just a vertical line $x=-\sigma / \beta=-1$ (for $\beta=\sigma$ ) in state space. The $x$ nullcline Eq. (11) is given by a cubic equation for $y$ as a function of $x$ :

$$
y=h(x)=x-\frac{\alpha}{1+x^{2}}
$$

The nullclines intersect at the no-noise fixed point. Figure 8 shows that the trajectories track the $x$ nullcline but make rapid transitions from one branch of the nullcline to the other at the local extrema. Standard phase-space methods [49] show that the recovery and pulse branches are stable in the sense that trajectories starting near those branches quickly approach those branches, while the middle branch, between the two extrema, is unstable.

In using the nullclines to define the trajectories, we are making two approximations. First, we ignore the time spent in making the transition from one branch of the nullcline to the other. Figures 3 and 8 show that the transition in the fast variable $x$ is not instantaneous, but for $\sigma=\beta=10^{-3}$, the transition time is less than $5-6 \%$ of the pulse and activation times. Second, the nullcline approximation ignores the oscillatory behavior of the trajectories in the neighborhood of the (no-noise) fixed point. We believe that this approximation is the limiting factor in our calculations. In order to use Eqs. (9) and (10), we need a potential function $U(y)$ for the dynamics. We now proceed to find $U(y)$.

From Eq. (12), we see that the deterministic part of the $y$ dynamics is given by

$$
y_{n+1}-y_{n}=-\beta x_{n}-\sigma .
$$

We then introduce the following ordinary differential equation (ODE):

$$
\dot{y}=-\beta x(t)-\sigma .
$$

In employing Eq. (15) in place of Eq. (14), we are assuming that the unit time step is sufficiently small compared to the activation, pulse, and recovery durations and that the changes in $y$ per unit time step are small (see Fig. 3) so that we may use $\dot{y} \Delta t=y_{n+1}-y_{n}$ with $\Delta t=1$. In other words, Eq. (14) can be viewed as an Euler approximation for the ODE Eq. (15).

We use $x$ as a function of $y$ on the $x$ nullcline to convert Eq. (15) into a differential equation involving $y$ alone. In principle, we could use Eq. (13) (a cubic equation) to solve for $x$ in terms of $y$ and then find $d U / d y$. We would then integrate that result to find $U(y)$ [9]. That procedure, however, leads to intractable first-exit-time integrals. In practice, it is simpler to approximate the nullcline near its extrema with quadratic functions. (Since the "escape" from one branch to the other occurs near the extrema, we need approximate the nullclines only near the extrema.) Let $\left(x_{e A}, y_{e A}\right)$ and $\left(x_{e P}, y_{e P}\right)$ be the coordinates of the two $x$ nullcline extrema. We approximate the nullcline in the neighborhood of the extrema as

$$
y=y_{e A, e P}+\frac{1}{2} \gamma_{A, P}\left(x-x_{e A, e P}\right)^{2},
$$

where $\gamma_{A}$ and $\gamma_{P}$ are parameters to be determined later. (For the Rulkov model we see that $\gamma_{P}>0$ for the pulse phase and $\gamma_{A}<0$ for the recovery-activation phase.) This approximation in fact works well along the entire recovery-activation and pulse branches visited by the trajectories. Solving for $x$ in terms of $y$ yields

$$
x=x_{e A, e P} \mp \sqrt{\frac{2\left(y-y_{e A, e P}\right)}{\gamma_{A, P}}},
$$

where the upper sign applies to the recovery and activation (left) branch and the lower sign to the pulse (right) branch of the $x$ nullcline.

The Langevin equation for the $y$ dynamics becomes

$$
\dot{y}= \pm \beta \sqrt{\frac{2\left(y-y_{e A, e P}\right)}{\gamma_{A, P}}}-\left(\beta x_{e A, e P}+\sigma\right)+\sqrt{D_{y}} \xi(t) .
$$

From Eq. (18), we see that the potential function is given by

$$
U_{A, P}(y)=\mp \beta \sqrt{\frac{2}{\gamma_{A, P}}} \frac{2}{3}\left(y-y_{e A, e P}\right)^{3 / 2}+\left(\beta x_{e A, e P}+\sigma\right) y \text {. }
$$

We may safely ignore additive constants in the potential function since they will cancel in the first-exit-time integrals. Since $\gamma_{A}$ is negative on the activation branch and $y-y_{e A}$ is also negative, we rewrite the previous equation as

$$
U_{A, P}(y)=\mp \beta \gamma_{A, P} \frac{\sqrt{8}}{3}\left|\frac{y-y_{e A, e P}}{\gamma_{A, P}}\right|^{3 / 2}+\left(\beta x_{e A, e P}+\sigma\right) y,
$$

where again the upper sign applies to the recovery branch and the lower to the pulse branch.

We determine the potential parameters $\gamma_{A, P}$ from the $x$ nullcline function $h(x)$ given in Eq. (13): 


$$
\gamma_{A, P}=\left.\frac{\partial^{2} h(x)}{\partial x^{2}}\right|_{x_{e A, e P}}=\left.\frac{2 \alpha}{\left(1+x^{2}\right)^{2}}\left[1-\frac{4 x^{2}}{1+x^{2}}\right]\right|_{x_{e A, e P}} .
$$

All of the relevant parameter values can be calculated directly from the Rulkov model equations.

In using the potential function to evaluate the regularity, it turned out to be useful to calculate the recovery and activation times separately even though they use the same $U(y)$. Since the integrands of the integrals used to evaluate the first-exit times are sharply peaked near the no-noise fixed point, the numerical procedures were simplified by treating the recovery and activation times separately, the latter of which is dominated by behavior near the fixed point.

Although we have reduced the calculation of the regularity to the numerical evaluation of integrals, we note that these multidimensional integrals are notoriously difficult to evaluate precisely [50] because the integrands are very sharply peaked for small noise variances. To see what precision is required, we note that the regularity $R$ can be written as

$$
R=\left(\frac{\left\langle T^{2}\right\rangle}{\langle T\rangle^{2}}-1\right)^{-1 / 2}
$$

or equivalently

$$
\frac{\left\langle T^{2}\right\rangle}{\langle T\rangle^{2}}=1+\frac{1}{R^{2}}
$$

If we want to calculate $R$ with a relative precision $\delta R / R$, we must be able to calculate the ratio of $\left\langle T^{2}\right\rangle$ to $\langle T\rangle^{2}$ with a precision $2 \delta R / R^{3}$. For example, if we wish to calculate a regularity value of 10 with $10 \%$ relative precision, the ratio of the mean values must be given to a precision of about $0.2 \%$. For small noise variances, this turns out to be quite tedious and computationally expensive, particularly for the integrals for $\left\langle T_{A}\right\rangle$ and $\left\langle T_{A}^{2}\right\rangle$, whose integrands are sharply peaked near the fixed point. In the Appendix, we develop an analytic approximation for low noise amplitudes that is computationally more efficient. The results of the analysis displayed in the figures, however, were calculated using the full numerical evaluation of Eqs. (9) and (10) with the appropriate potential functions.

In using Eqs. (9) and (10) to fit the data, we made small changes in the location of the escape point (the absorbing boundary) of the activation phase to compensate for the neglect of the oscillatory motion near the fixed point. [An expanded version of Fig. 8 (not shown) indicates that the escape point is not precisely at the extremum of the $x$ nullcline.] The fractional shift in the escape point was typically $10^{-3}-10^{-4}$. There are no other adjustable parameters. Figure 9 shows the results of the analysis for $\beta=\sigma=0.001$ and two values of $\alpha$. The agreement with the simulations is remarkably good given the simplicity of the models of the dynamics.

$$
\text { E. } D_{y}=0
$$

In this section we treat the case when noise is present only in the dynamics of the fast $(x)$ variable. Because we are now

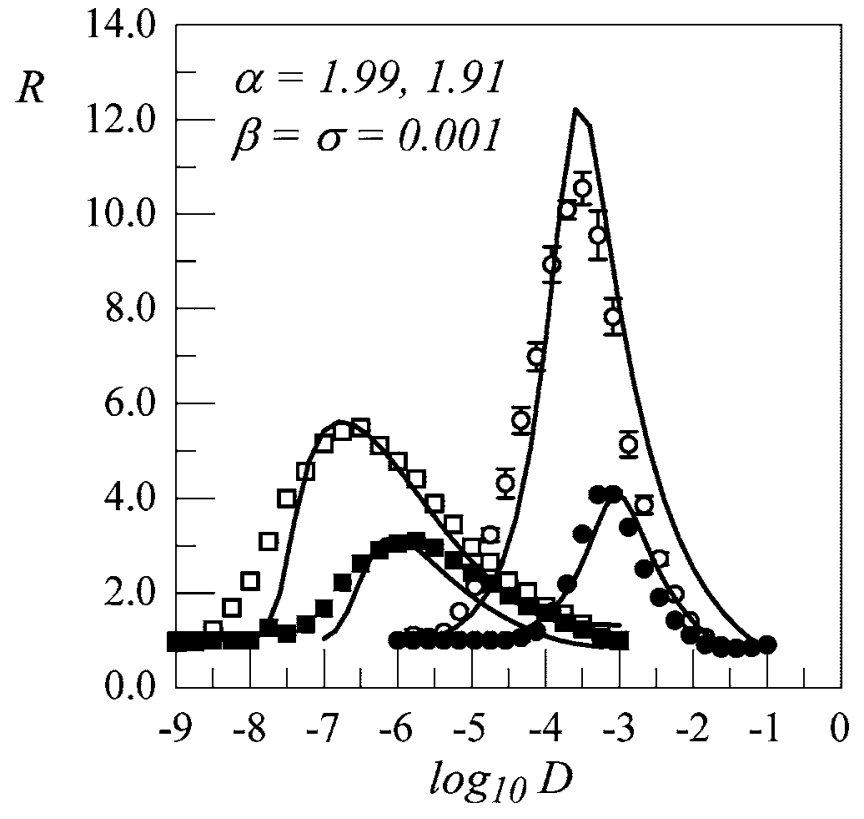

FIG. 9. The results of the Fokker-Planck analysis described in the text (solid curves) and the simulation data for the Rulkov model with $\beta=\sigma=10^{-3}$. Open symbols, $\alpha=1.99$; solid symbols, $\alpha=1.91$. On the left $D_{x}=0$ (squares); on the right $D_{y}=0$ (circles).

focusing on the dynamics of the fast variable, we can no longer use the adiabatic elimination procedure to reduce the system to a one-dimensional system. Instead, we proceed phenomenologically and simply assume one-dimensional behavior. Again, we divide the dynamics into three phases: an activation phase in the neighborhood of the no-noise fixed point, a pulse phase, and a recovery phase. The activation phase can be handled by standard procedures because the behavior can be described as fluctuations around a fixed point of the system. In particular, for the activation phase, we assume that $U(x)$ is a one-dimensional quadratic potential $U(x)=\frac{1}{2} k_{A}\left(x-x^{*}\right)^{2}$ where $x^{*}$ is the no-noise fixed point. We determine the parameter $k_{A}$ by evaluating the Jacobian determinant of the map functions Eqs. (1) and (2) at the no-noise fixed point.

For a two-dimensional map system, we have two (local) Lyapunov exponents, which in general may be complex. For the Rulkov map model, the Jacobian matrix evaluated at the fixed point $\left(x^{*}, y^{*}\right)$ is given by

$$
\left(\begin{array}{cc}
\left.\frac{\partial f}{\partial x}\right|_{*} & \left.\frac{\partial f}{\partial y}\right|_{*} \\
\left.\frac{\partial g}{\partial x}\right|_{*} & \left.\frac{\partial g}{\partial y}\right|_{*}
\end{array}\right)=\left(\begin{array}{cc}
\frac{-2 \alpha x^{*}}{\left(1+x^{* 2}\right)^{2}} & 1 \\
-\beta & 1
\end{array}\right) .
$$

At the fixed point, we have $x^{*}=-1$ (for $\left.\beta=\sigma\right)$, and the eigenvalues are

$$
\Lambda_{1,2}=\frac{\alpha+2 \pm \sqrt{(\alpha-2)^{2}-16 \beta}}{4} .
$$

With $\beta=0.001$ and $1.998<\alpha<1.999$, the two eigenvalues form a complex conjugate pair, the absolute value of the 
eigenvalues exceeds 1 , and the behavior displays sustained oscillations. Near the lower limit of this range, the oscillations are nearly sinusoidal and centered approximately on the fixed point. At the upper end of this range the oscillations are non sinusoidal. For $\alpha<1.998$, the range relevant for our considerations, the approach to the fixed point consists of exponentially damped oscillations (see Fig. 2). In the standard way [51], we express the two local Lyapunov exponents $\lambda_{1}$ and $\lambda_{2}$ in terms of the eigenvalues $\Lambda_{1}$ and $\Lambda_{2}$ :

$$
\lambda_{1}=\ln \Lambda_{1}, \quad \lambda_{2}=\ln \Lambda_{2} .
$$

(The real part of the Lyapunov exponents determines the divergence or convergence of nearby trajectories.)

For our one-dimensional model, we ignore the oscillations and focus on the exponential decay to the fixed point. The potential parameter $k_{A}$ is related to the local Lyapunov exponent in the usual way [51]:

$$
k_{A}=-\operatorname{Re}(\lambda)=-\ln |\Lambda| .
$$

As $\alpha$ decreases, $k_{A}$ increases, indicating a larger barrier to surmount before escape from the fixed point region.

To use Eqs. (9) and (10) for the activation phase, we also need numerical values for the absorbing boundary location relative to the fixed point, the injection point, and the reflecting boundary. The injection point is taken to be the no-noise fixed point. The absorbing boundary was chosen to give a reasonable match to the mean activation time as a function of the noise amplitude. The results were insensitive to the location of the reflecting boundary.

For the pulse and recovery phases, the system's behavior remains close to the (no-noise) fixed points of Eq. (1) with $y$ viewed as a slowly changing parameter. In general, there will be three fixed points. See Fig. 8. First, let us consider the pulse phase. We assume that the fixed point $x_{3}^{*}$ near $x=0$ moves at a constant "velocity" $v_{P}$ during the pulse phase. With this approximation, in the absence of noise, the pulse phase time is given by

$$
T_{P}=\frac{a_{P}-w_{P}}{v_{P}},
$$

where $a_{P}-w_{P}$ is the "distance" traveled by the fixed point from the injection point $w_{P}$ to the absorbing point $a_{P}$ at the rate given by $v_{P}$. When noise is present, we get fluctuations in the pulse time due to the noise. We model the fluctuations by adding a fluctuating term $\varepsilon$ to the distance traveled during the pulse phase:

$$
T_{P}(\varepsilon)=\frac{a_{P}-w_{P}+\varepsilon}{v_{P}} .
$$

The mean pulse length is then given by

$$
\left\langle T_{p}\right\rangle=\frac{a_{P}-w_{P}}{v_{P}},
$$

since the mean of the noise term is zero.

The noise, however, does affect the mean-squared pulse time. The mean-squared pulse time is given by

$$
\left\langle T_{P}^{2}\right\rangle=\left\langle\left[\left(a_{P}-w_{P}+\varepsilon\right) / v_{P}\right]^{2}\right\rangle=\left(\frac{a_{P}-w_{P}}{v_{P}}\right)^{2}+\frac{\left\langle\varepsilon^{2}\right\rangle}{v_{P}^{2}} .
$$

To relate the fluctuations to the noise variance $D$, we assume that the behavior around the (moving) fixed point can be modeled as an Ornstein-Uhlenbeck process for which the associated Langevin equation is

$$
\dot{\varepsilon}=-k_{P} \varepsilon+\sqrt{D} \xi(t) .
$$

Under these conditions, the variance of $\varepsilon$ is time-dependent $[52,53]$ :

$$
\operatorname{var}(\varepsilon)=\frac{D^{2}}{2 k_{P}}\left(1-e^{-2 k_{P} t}\right) .
$$

$k_{P}$ is determined from the local Lyapunov exponent at the fixed point $x_{3}^{*}$. In the Rulkov model, however, the Lyapunov exponent varies with $x$ in the pulse phase. Hence, we use an average value weighted toward the smaller values of $x$ just before the transition to the recovery phase. The "velocity" $v_{P}$ can be estimated from the pulse duration. See Figs. 2 and 3. As an example, for $\alpha=1.99$ and $\beta=\sigma=10^{-3}$, we find that $k_{P} \approx 0.2$ and $T_{P} \approx 100$ for the Rulkov model with $\beta=\sigma$ $=10^{-3}$. Hence, we are in the long-time limit, and Eq. (33) reduces to

$$
\left\langle\varepsilon^{2}\right\rangle=\frac{D^{2}}{2 k_{P}} .
$$

Using Eq. (34) in Eq. (31), we find that the mean-squared pulse time is given by

$$
\left\langle T_{P}^{2}\right\rangle=\left(\frac{a_{P}-w_{P}}{v_{P}}\right)^{2}+\frac{D^{2}}{2 k_{P} v_{P}^{2}},
$$

where again the angular brackets indicate an average over noise realizations.

We use a similar analysis for the recovery phase, where the trajectory tracks the fixed point $x_{1}^{*}$ near $x \approx-1.5$, with the results

$$
\left\langle T_{R}\right\rangle=\frac{a_{R}-w_{R}}{v_{R}}
$$

and

$$
\left\langle T_{R}^{2}\right\rangle=\left(\frac{a_{R}-w_{R}}{v_{R}}\right)^{2}+\frac{D^{2}}{2 k_{R} v_{R}^{2}} .
$$

For the recovery and pulse phases, the injection point $w$ and the absorbing point $a$ are determined from the nullcline.

Figure 9 shows the data and the results of our calculations for the regularity as a function of noise for two values of $\alpha$. The adjustable parameters are the absorbing boundary (which we allow, as before, to be slightly different from the extremum of the $x$ nullcline) for the activation phase and the parameters $k_{P}$ and $k_{R}$ for the pulse and recovery phases. We see that the agreement is quite good given the simplicity of the approximations. 


\section{F. Two noise sources}

When both fast and slow noise sources are present or if we wish to go beyond the one-dimensional approximations used above, we need to develop a full two-dimensional analysis of the first-exit time problem. In this paper, we will use a simple statistical argument to link the two onedimensional models. We shall see that this simple method gives a reasonable account of the data shown in Fig. 6.

In the low-noise limit, each noise source alone produces a series of pulses with the number of pulses in a given time interval described by a Poisson distribution. As is well known [42], the corresponding interspike intervals have an exponential distribution. The mean interspike interval is determined primarily by the activation time, and the mean activation time is given by the reciprocal of the activation rate $\left\langle T_{A}\right\rangle=1 / r$ and the variance is given by $\operatorname{var}\left(T_{a}\right)=1 / r^{2}$.

If both noise sources are present, in the low-noise limit the activation rate $r$ will be the sum of the activation rates of the two sources individually. In that case the mean activation time is given by

$$
\left\langle T_{A}\right\rangle=\frac{\left\langle T_{f}\right\rangle\left\langle T_{s}\right\rangle}{\left\langle T_{f}\right\rangle+\left\langle T_{s}\right\rangle},
$$

where $T_{f, s}$ are the activation times due to each of the noise sources (fast and slow) individually. Similarly, the meansquared activation time is given by

$$
\left\langle T_{A}^{2}\right\rangle=\frac{\left\langle T_{f}^{2}\right\rangle\left\langle T_{s}^{2}\right\rangle}{2\left(\sqrt{T_{f}^{2} / 2}+\sqrt{T_{s}^{2} / 2}\right)^{2}} .
$$

It is straightforward to show that if $T_{f}$ and $T_{s}$ have exponential distributions, so does $T_{A}$. Given the mean activation time and mean-square activation time for each of the noise sources individually, we can use Eqs. (38) and (39) to find the corresponding quantities for the overall activation time.

For the pulse and recovery phases, we use the minimum of $\left\langle T_{P, R}\right\rangle$ for either the fast or slow noise to characterize the times for those phases of the dynamics. In essence, whichever process (fast noise or slow noise) gets the system to the end of the pulse phase or the recovery phase first wins. Figure 10 shows the results of this simple statistical analysis for the Rulkov model with the means and variances calculated as described in Secs. IV D and IV E. The results are in reasonably good agreement with the simulation data shown in Fig. 6.

\section{FITZHUGH-NAGUMO MODEL AND STOCHASTIC COHERENCE}

In this section we turn to a continuous-time, differential equation model of an excitable neuron. Neuron behavior has traditionally been described by differential equation models more closely tied to physiological processes than is the Rulkov discrete-time model. We will use the well-known FitzHugh-Nagumo model [54,55]. We employ the form used by [9] but with independent noise terms added to each equation:

$$
\frac{d v}{d t}=\frac{1}{\varepsilon}\left[v(t)-v^{3}(t)-w(t)\right]+\sqrt{D_{v}} \eta_{v}(t)
$$

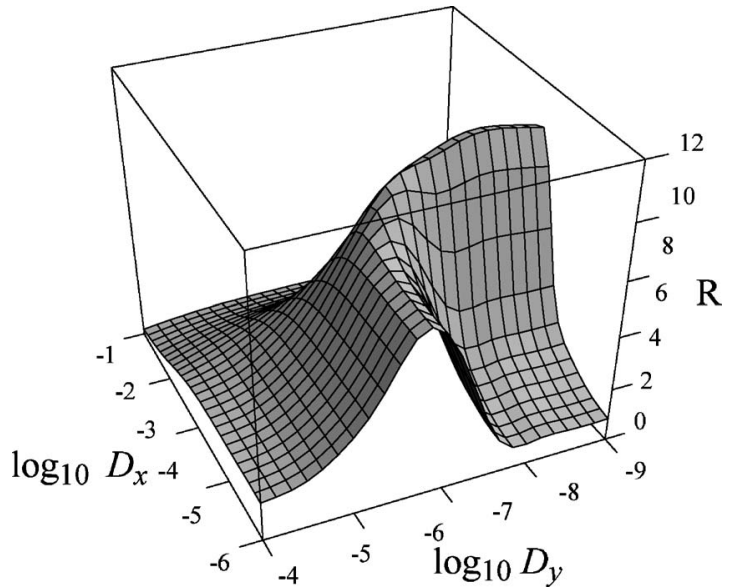

FIG. 10. The results of the Fokker-Planck analysis of the Rulkov model stochastic coherence with noise sources present in both dynamical variables. $\alpha=1.99$ and $\beta=\sigma=10^{-3}$. The regularity $R$ is plotted as a function of $\log _{10} D_{x}$ and $\log _{10} D_{y}$. Note the general agreement with the simulation results presented in Fig. 6.

$$
\frac{d w}{d t}=\gamma v(t)-w(t)+b+\sqrt{D_{w}} \eta_{w}(t) .
$$

Here $v$ represents the neuron membrane voltage and $w$ describes a gating-ion concentration. $\varepsilon$ is a small parameter that sets the separation of the fast and slow time scales. We shall use $\varepsilon=0.001$ in our analysis. The parameters $\gamma$ and $b$ set the operating conditions for the model. We shall use $\gamma=1.5$. For $b<0.481125$, the model exhibits periodic pulses. To exhibit stochastic coherence in this model, we shall use $b=0.4812$ and 0.53 , both of which put the model in an excitable regime. The two independent Gaussian-distributed noise sources $\eta_{v}$ and $\eta_{w}$ have zero mean and variance $D$.

We integrated Eqs. (40) and (41) using a modified Euler procedure as described by Gillespie [52]. The integration step size was chosen to be small enough to reproduce accurately the no-noise pulse behavior (for $b<0.481125$ ) generated by a high-precision adaptive Runge-Kutta method. Figure 11 displays the regularity of the noise-induced pulses as a function of the logarithm of the noise variance for the FitzHugh-Nagumo model. Again we see that the noise added to the slow dynamics produces a regularity with a smaller maximum at lower noise amplitudes than does noise added to the fast dynamics.

\section{FOKKER-PLANCK ANALYSIS OF FITZHUGH- NAGUMO STOCHASTIC COHERENCE}

We can provide a quantitative account of stochastic coherence in the FitzHugh-Nagumo model in analogy with our analysis for the Rulkov model. For the parameter values used here and in the absence of noise, the system approaches the (no-noise) fixed point given by the intersection of the $v$ and $w$ nullclines. When a sufficiently large noise burst kicks the system away from the fixed point, the trajectories follow the stable branches of the $v$ nullcline until a jump occurs between branches. Figure 12 shows the $v$ and $w$ nullclines for 


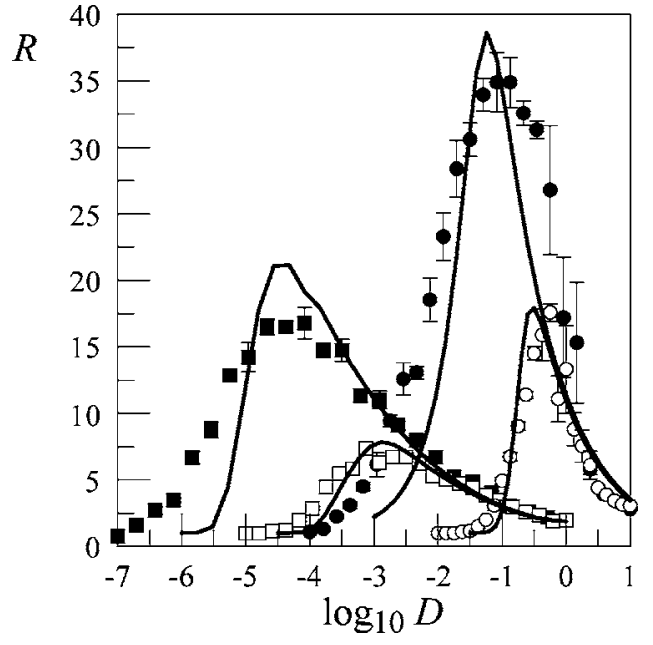

FIG. 11. A plot of the regularity of the noise-induced pulses for the FitzHugh Nagumo model. $\varepsilon=0.001, \gamma=1.5$. Solid symbols, $b$ $=0.4812$; open symbols, $b=0.53$; squares, $D_{v}=0$; circles, $D_{w}=0$. The uncertainty bars indicate the standard deviation of the mean for ten noise realizations, each consisting of a sequence of 200 time units. (Each pulse has a duration of about 1.5 time units.) The solid curves are the results of the Fokker-Planck analysis described in the text.

the FitzHugh-Nagumo (FHN) model superposed on several noise-induced pulse trajectories.

For noise added only to the slow $w$ variable, we can use adiabatic elimination of the voltage variable $v$ to find an effective potential for the $w$ dynamics. That is, we need to solve for $v(w)$ along the $v$ nullcline. This solution can be found exactly [9] using $w=v-v^{3}$, but as with the Rulkov model, it is computationally simpler to use a quadratic ap-

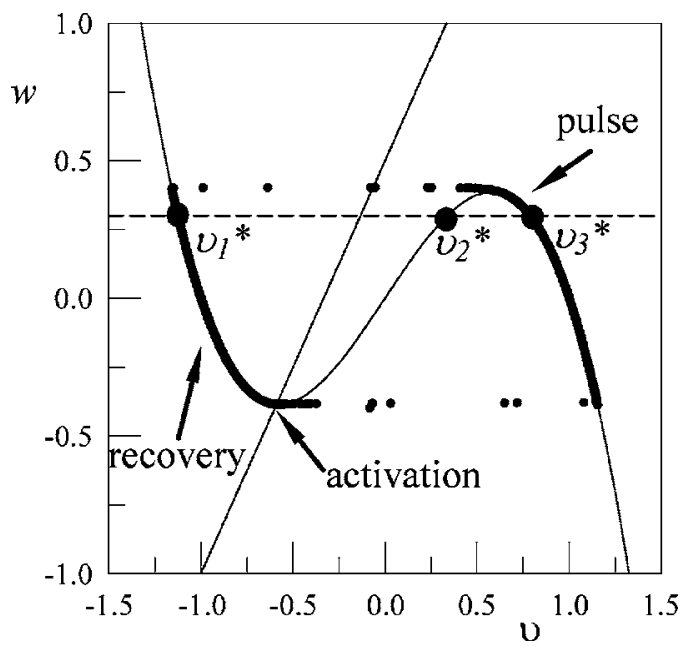

FIG. 12. $v$ nullcline (cubic curve) and $w$ nullcline (straight line) for the FitzHugh-Nagumo model superposed on several noiseinduced pulse trajectories (small circles) for $b=0.4812, \gamma=1.5$, and $\varepsilon=10^{-3}$. The activation, pulse, and recovery phases are indicated. The coordinates of the $v$ nullcline local minimum are $\left(v_{A}, w_{A}\right)$ and those of the local maximum are $\left(v_{P}, w_{P}\right)$. The three fixed points for the no-noise version of Eq. (40) are indicated by the larger, filled-in circles. proximation near the extrema of the nullcline. Near the extrema at $\left(v_{A, P}, w_{A, P}\right)$, we may write

$$
w(v)=w_{A, P}+\frac{1}{2} k_{A, P}\left(v-v_{A, P}\right)^{2} .
$$

Taking the second derivative of the $v$ nullcline equation, we find that $k_{A, P}=-6 v_{A, P}$. Solving for $v$ gives us

$$
v=v_{A, P} \mp \sqrt{\frac{2\left(w-w_{A, P}\right)}{k_{A, P}}},
$$

where the upper sign applies to the activation-recovery branch and the lower sign to the pulse branch. Since the deterministic part of the Langevin equation for $w(t)$ is given by

$$
\frac{d w}{d t}=\gamma v-w+b=\gamma v_{A, P} \mp \gamma \sqrt{\frac{2\left(w-w_{A, P}\right)}{k_{A, P}}}-w+b,
$$

we see that the effective potentials for the activationrecovery and pulse phases are given by

$$
U_{A, P}(w)= \pm \frac{\sqrt{8}}{3} \gamma k_{A, P}\left(\frac{w-w_{A, P}}{k_{A, P}}\right)^{3 / 2}-w\left(\gamma v_{A, P}+b\right)+w^{2} / 2,
$$

where again the upper sign applies to the activation-recovery phase and the lower sign to the pulse phase.

Equation (45) may now be used in Eqs. (9) and (10) to find the mean activation and pulse times as well as their variances. (In practice, we separate the recovery and activation phases as we did for the Rulkov model.) From those quantities we calculate the regularity for the FHN model for the case in which noise is added only to the slow variable $w$. The results are shown in Fig. 11. The only adjustable parameter is the activation-phase absorbing boundary, which is close to the $v$ nullcline local minimum. The typical fractional deviation from the nullcline minimum is $10^{-3}$.

When noise is added only to the fast variable $v$, we use a method identical to that used in the Rulkov model: we assume that the dynamics track the (no-noise) fixed points of Eq. (40) during the pulse and recovery phases with $w$ viewed as a slowly changing parameter. The fixed points $v^{*}$ satisfy the equation

$$
v^{*}-v^{* 3}=w,
$$

yielding, in general, three fixed points. During the recovery and activation phase, the system tracks the fixed point $v_{1}^{*}$ shown in Fig. 12. Eventually $w$ decreases sufficiently and $v_{1}^{*}$ disappears. Then the system jumps to the fixed point $v_{3}^{*}$ in Fig. 12 corresponding to the pulse phase. $w$ then increases until $v_{3}^{*}$ disappears and the system jumps back to $v_{1}^{*}$.

For the pulse and recovery phases, we model the dynamics near the fixed point $v^{*}$ as motion in a quadratic potential

$$
U_{P, R}(v)=\frac{1}{2} \lambda_{P, R}\left(v-v_{3,1}{ }^{*}\right)^{2},
$$

where $\lambda_{P, R}=\left.(d G / d v)\right|_{v_{3,1}^{*}}$ and $G(v)=\left(v-v^{3}-w\right) / \varepsilon$. In analogy with the Rulkov model analysis for the pulse and recovery phases, we have 


$$
\left\langle T_{P, R}\right\rangle=\frac{a_{P, R}-w_{P, R}}{v_{P, R}}
$$

and

$$
\left\langle T_{P, R}^{2}\right\rangle=\left\langle T_{P, R}\right\rangle^{2}+\frac{D_{v}}{2 \lambda_{P, R} v_{P, R}^{2}},
$$

where $v_{P, R}$ is the velocity of the fixed point during the pulse or recovery phase, respectively. Since both $\lambda_{P}$ and $\lambda_{R}$ change as the fixed point moves, we use values weighted to emphasize the noise dependence near the end of the pulse and recovery phases.

For the activation phase, we use a quadratic potential model $U_{A}(v)=\frac{1}{2} k_{A}\left(v-v_{0}^{*}\right)^{2}$ with the potential parameter $k_{A}$ determined by the eigenvalues of the Jacobian matrix of the FitzHugh-Nagumo equations (40) and (41). $v_{0}^{*}$ is the no-noise fixed point of the FitzHugh-Nagumo equations. The matrix takes the form

$$
J^{*}=\left(\begin{array}{cc}
\left(1-3 v_{0}^{* 2}\right) / \varepsilon & -1 / \varepsilon \\
\gamma & -1
\end{array}\right) .
$$

Figure 11 shows the results of this analysis. Again, the agreement with the simulation data is satisfactory given the simple nature of the approximations used. The only adjustable parameters were the location of the absorbing boundary for the activation phase and the parameters $\lambda_{P, R}$ for the pulse and recovery phases.

\section{DISCUSSION AND CONCLUSIONS}

We have shown that relatively simple one-dimensional models of the dynamics can account for the fast and slow noise effects in two distinct models of excitable systems. For both models, we explored the effects of changing several of the parameters. We now give a more qualitative explanation of the observed effects and draw some general conclusions about our approach.

First, let us discuss how the Rulkov model results depend on the parameters of the model. As $\alpha$ decreases below 1.99, the no-noise fixed point moves further from the nullcline extremum, thereby increasing the barrier the system must overcome before it can move away from the fixed point. Hence, the noise variance must be larger to achieve a given level of activation. This effect explains the shift in the location of the regularity maximum as $\alpha$ decreases as illustrated in Fig. 5. Since the noise variance is larger, the noise has a larger effect on the pulse and recovery times, thus lowering the overall regularity of the pulse sequence as seen in Fig. 5 for smaller values of $\alpha$. A similar effect occurs with increasing $b$ in the FitzHugh-Nagumo model as illustrated in Fig. 11.

When we change the parameters $\sigma$ and $\beta$ in the Rulkov model (restricting ourselves to the case $\sigma=\beta$ ), we are effectively changing the separation between the fast and slow time scales. Comparing Figs. 5 and 7, we see that for smaller values of $\sigma$ and $\beta$, when the time scale separation is larger, the distinction between noise added to the fast and slow dynamics is enhanced. In that case, the slow variable dynamic

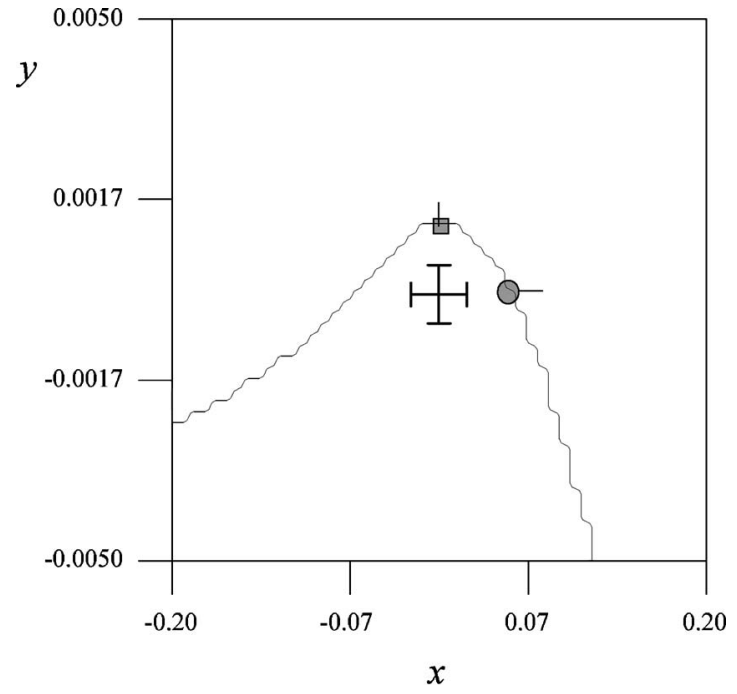

FIG. 13. A plot of the escape region for the no-noise Rulkov model for $\alpha=1.99$ and $\beta=\sigma=0.001$. Initial points below the curve lead to trajectories that stay in the neighborhood of the fixed point, located at $(0,0)$ in this plot. (The steps in the curve are due to finite sampling of the initial conditions.) Initial points above the curve lead to trajectories that make a large excursion through state space before returning to the fixed point. The cross, centered on the fixed point, indicates the standard deviation of the noise intensity when the regularity has its maximum value for these parameter values. The filled circle and square indicate the escape points for the onedimensional models. The short vertical and horizontal lines indicate the range of escape points used in the data fits.

range is reduced compared to that of the fast variable and smaller values of the noise variance in the slow dynamics are required to achieve activation. Conversely, increasing $\beta$ and $\sigma$ reduces the time scale separation and the difference in the regularity with fast and slow noise is reduced. Reference [9] demonstrated a similar, but less dramatic, shift in the regularity for the case in which noise is added only to the slow dynamics in the FitzHugh-Nagumo model when the time scale separation was changed.

We can look at these effects another way by determining which initial conditions in the neighborhood of the no-noise fixed point lead to trajectories that escape from the fixed point and which stay in its neighborhood. Figure 13 illustrates the escape region for the Rulkov model for $\alpha=1.99$ and $\beta=\sigma=10^{-3}$. We see that deviations from the fixed point required to generate a pulse are much smaller in the $y$ (slow variable) direction than in the $x$ (fast variable direction). The bars centered on the fixed point location indicate the standard deviation of the noise intensity when the regularity has its maximum value. Compare Fig. 5. We should note that the trajectories may oscillate around the fixed point before escaping. Nevertheless, Fig. 13 gives an indication of the relative sizes of the deviations from the fixed point required to generate a pulse. The difference in ranges for $x$ and $y$ are consistent with the difference between the relevant noise ranges as illustrated in Figs. 5 and 7. We emphasize that Fig. 13 is a result of the simulations and that all of the results of the Fokker-Planck analyses were carried out with the onedimensional models described in Secs. IV and VI. 
When $\beta$ and $\sigma$ increase, the boundaries of the escape region are further from the fixed point (not shown) and a larger noise variance is required for activation. This accounts for the shift towards larger $\log D$ for larger $\beta$ and $\sigma$ seen in Fig. 7. The larger noise variance is then more disruptive for the pulse and recovery phases leading to lower maximum values of the regularity.

We have seen that the maximum value for the regularity is larger when noise is added to the fast variable dynamics than when it is added to the slow variable dynamics. The fast variable behavior is linked to the fixed points, whose locations drift as the slow variable evolves. Hence, the noise is less disruptive of the dynamics when added to the fast variable because of the "restoring" force associated with the fixed points. On the other hand, the slow variable dynamics, except for the behavior near the no-noise fixed point, is associated with "drift" motion along the nullcline, for which there is no restoring force. The drift motion is more easily disturbed by noise and hence the regularity is reduced compared to the fast dynamics case.

When noise is present for both the fast and slow dynamics, we see that stochastic coherence occurs as a function of $D_{y}$, only if $D_{x}$ is sufficiently small. See Fig. 6. If $D_{x}$ is near to or larger than the value at which the maximum in the regularity occurs for $x$ noise alone, the $y$ noise just attenuates the maximum regularity. Conversely, if $D_{y}$ is sufficiently large, there will be no maximum in the regularity viewed as a function of $D_{x}$. The practical implication is that a small amount of noise in the slow variable can wipe out the stochastic coherence maximum as a function of noise intensity in the fast variable. Thus experimenters looking for stochastic coherence in systems with multiple time scales must be careful to minimize the noise in the slow variable(s) to permit observation of stochastic coherence as a function of noise in the dynamics of the fast variable(s). (Since the relevant noise range for the fast variable is much larger than that for the slow variable, we assume that the experimenter will be aware of noise added to the fast variable.)

We have demonstrated that a one-dimensional FokkerPlanck first-exit-time analysis leads to predictions for the regularity as a function of noise intensity that are in reasonably good agreement with the results of the numerical simulations. There are, however, significant differences between the Fokker-Planck results and the simulations, particularly for low noise amplitudes. Several factors can account for the deviations. First, and perhaps the most important, is our neglect of the two-dimensional oscillatory dynamics in the neighborhood of the fixed point. Our one-dimensional models by necessity miss that oscillatory behavior. When the systems are further from threshold, the large noise intensity required to activate the system masks the oscillatory behavior and the agreement with the numerical simulations is enhanced.

A second deficiency in our models is the neglect of the dynamics in the transitions from the fixed point to the pulse phase and from the pulse phase to the recovery phase. The transition times become more important for larger values of $\beta$ and $\sigma$ and Fig. 7 shows that the Fokker-Planck results are less satisfactory for $\beta=\sigma=10^{-2}$. Third, we have approximated the pulse and recovery phases using rather simple models that miss some of the dynamics near the transition points. In spite of these limitations, the general agreement between the simulation results and the Fokker-Planck analysis is rather good given that the noise range extends over five or six orders of magnitude for most of the results. This agreement indicates that we have accounted for the essential physics of the fast and slow noise effects.

Although we made no attempt to model the behavior of actual neurons, we note that the fast dynamics noise levels explored in this paper are consistent, within an order of magnitude, with the voltage noise levels observed in real neurons [56]. Ion concentration fluctuations are more difficult to model. Furthermore, on the physiological side, ion fluctuations vary considerably among different types of neurons and depend on conditions in the neuron itself. Nevertheless, the noise levels required for stochastic coherence presented here are consistent with (within an order of magnitude) "typical" calcium-concentration fluctuations $[57,58]$. The calciumconcentration fluctuations, however, vary widely due to clustering of release mechanisms and the production of spatial waves of calcium within the cell.

We argue that the fast and slow noise effect demonstrated and explained here is quite general and should occur for any excitable system with multiple time scales. Recognizing this effect should also be important in experimental studies of stochastic coherence. Specifically, if low-level (and perhaps barely detectable) noise is present in the dynamics of the slow variable, stochastic coherence for the fast variable dynamics may be significantly reduced or even eliminated. Where possible, experimentalists should vary the noise intensity for both the fast and slow dynamics.

\section{ACKNOWLEDGMENTS}

We thank W. Loinaz for helpful discussions. R.C.H. thanks the Center for Theoretical Biological Physics and the Institute for Nonlinear Science, both at University of California at San Diego, where part of this work was completed.

\section{APPENDIX}

Here we derive an expression for the mean activation time of an excitable system, applicable in the limit of low noise. The result derived here is analogous to the well-known Kramers result [43] but differs from the Kramers result because the "escape" from the fixed point does not occur at a maximum of the potential function. Figure 14 shows a sketch of the potential function and the absorbing boundary applicable to both the Rulkov and FitzHugh-Nagumo models.

We repeat here the integral for the mean activation time:

$$
\left\langle T_{A}\right\rangle=\frac{2}{D} \int_{w}^{a} d u \int_{b}^{u} d v e^{2(U(u)-U(v)) / D} .
$$

For small noise variances, the most important contribution to the integral occurs near the fixed point $y^{*}$. The argument of the exponential will be largest when $u \sim a$ and $v \sim y^{*}$. We use, therefore, a double Taylor series expansion for the potential difference: 


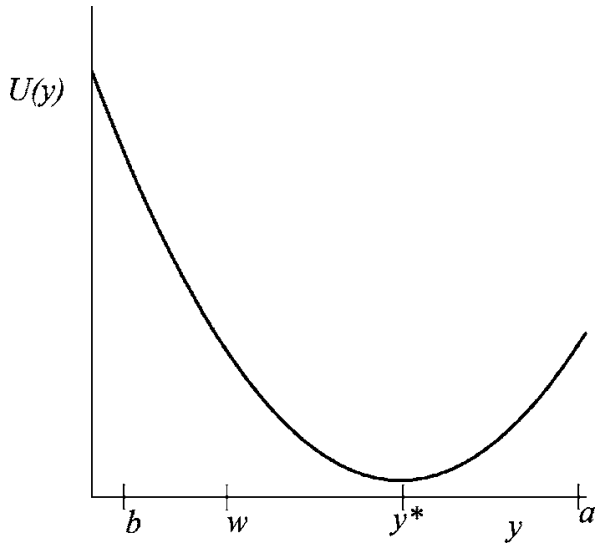

FIG. 14. A sketch of the potential function $U(y)$ indicating the absorbing boundary at $a$, the reflecting boundary at $b$, and the fixed point at $y^{*} . w$ is the injection point.

$$
\begin{aligned}
g(u, v)= & U(u)-U(v) \\
= & g\left(a, y^{*}\right)+\left.(u-a) \frac{\partial g}{\partial u}\right|_{a, y^{*}}+\left.\left(v-y^{*}\right) \frac{\partial g}{\partial v}\right|_{a, y^{*}} \\
& +\left.(u-a)\left(v-y^{*}\right) \frac{\partial^{2} g}{\partial u \partial v}\right|_{a, y^{*}}+\left.\frac{1}{2}(u-a)^{2} \frac{\partial^{2} g}{\partial u^{2}}\right|_{a, y^{*}} \\
& +\left.\frac{1}{2}\left(v-y^{*}\right)^{2} \frac{\partial^{2} g}{\partial v^{2}}\right|_{a, y^{*}}+\cdots .
\end{aligned}
$$

For the potential shown in Fig. 14, the linear $u$ dependence will dominate over the quadratic term. On the other hand, $g$ shows a maximum as a function of $v$ at $v=y^{*}$; so there only the quadratic term is important. We define two parameters that characterize the potential:

$$
\begin{gathered}
\left.\frac{\partial g}{\partial u}\right|_{a, y^{*}}=\left.\frac{\partial U}{\partial u}\right|_{a, y^{*}} \equiv c, \\
\left.\frac{\partial^{2} g}{\partial v^{2}}\right|_{a, y^{*}}=-\left.\frac{\partial^{2} U}{\partial v^{2}}\right|_{a, y^{*}} \equiv k<0 .
\end{gathered}
$$

Thus the approximate potential difference is

$$
\begin{aligned}
g(u, v) & \approx g\left(a, y^{*}\right)+(u-a) c-\frac{1}{2}|k|\left(v-y^{*}\right)^{2} \\
& \approx U(a)-U\left(y^{*}\right)+(u-a) c-\frac{1}{2}|k|\left(v-y^{*}\right)^{2} .
\end{aligned}
$$

First, we evaluate the $v$ integral

$$
I_{v}(u)=\int_{b}^{u} d v e^{-(|k| / D)\left(v-y^{*}\right)^{2}} .
$$

Assuming that $|k|\left(b-y^{*}\right) / D \gg 1$, which will certainly be true for small $D$, we can set the lower limit of the integral to $-\infty$.
We then break the integral into two parts, and use the change of variable $y=\sqrt{|k| / D}\left(v-y^{*}\right)$ to obtain

$$
I_{v}(u) \approx \sqrt{\frac{D}{|k|}}\left(\int_{-\infty}^{0} e^{-y^{2}} d y+\int_{0}^{\sqrt{|k| / D}\left(u-y^{*}\right)} e^{-y^{2}} d y\right) .
$$

The first integral in Eq. (A7) is equal to $\sqrt{\pi} / 2$. The second integral is $\sqrt{\pi} / 2$ times the error function

$$
\operatorname{Erf}(a)=\frac{2}{\sqrt{\pi}} \int_{0}^{a} e^{-y^{2}} d y
$$

The term in parentheses in Eq. (A7) has a lower limit of $\sqrt{\pi} / 2$ and an upper limit (for small noise values) of $\sqrt{\pi}$. For the purposes of approximation, we can set the parenthetic term equal to $\sqrt{\pi}$. The power series expansion of the error function could be used for a higher-order approximation.

We now tackle the $u$ integral. Taking into account the $v$ integral, we have

$$
\left\langle T_{A}\right\rangle \approx \frac{2 \sqrt{\pi}}{D} \sqrt{\frac{D}{|k|}} e^{(2 / D)\left[U(a)-U\left(y^{*}\right)\right]} e^{-2 c a / D} \int_{w}^{a} d u e^{2 c u / D} .
$$

The $u$ integral is straightforward and we obtain

$$
\left\langle T_{A}\right\rangle \approx \frac{\sqrt{\pi}}{c} \sqrt{\frac{D}{|k|}} e^{2 \Delta U / D}\left(1-e^{-2 c(a-w) / D}\right),
$$

where $\Delta U=U(a)-U\left(y^{*}\right)$. The parameters $c,|k|$, and $\Delta U$ can all be estimated from the potential function for the particular model being used. For the injection point, we may use $w$ $=y^{*}$.

Equation (A10) has some similarities to the standard Kramers expression $\langle T\rangle=T_{o} e^{\Delta U / D}$, but we see that the noise dependence here is a bit more complicated. This complication arises because the absorbing boundary is not a maximum in the potential function, which is the usual assumption made in deriving the Kramers formula.

We have found that Eq. (A10), along with the assumptions that the activation times are described by an exponential distribution and that the pulse and recovery phases are unaffected by noise, gives a good account of the low noise behavior of our simulations. With these assumptions the regularity is given by

$$
R=\frac{\left\langle T_{A}\right\rangle+\left\langle T_{R}\right\rangle+\left\langle T_{P}\right\rangle}{\sqrt{\operatorname{var}\left(T_{A}\right)}} .
$$

Equation (A11) reproduces the results of the full analysis quite well for low noise variances while being considerably faster computationally. 
[1] L. Gammaitoni, P. Hänggi, P. Jung, and F. Marchesoni, Rev. Mod. Phys. 70, 223 (1998).

[2] Hu Gang, T. Ditzinger, C. Z. Ning, and H. Haken, Phys. Rev. Lett. 71, 807 (1993).

[3] A. Longtin, Phys. Rev. E 55, 868 (1997).

[4] A. S. Pikovsky and J. Kurths, Phys. Rev. Lett. 78, 775 (1997).

[5] A. Zaikin and J. Kurths, Chaos 11, 570 (2001).

[6] S.-G. Lee, A. Neiman, and S. Kim, Phys. Rev. E 57, 3292 (1998).

[7] J. L. A. Dubbeldam, B. Krauskopf, and D. Lenstra, Phys. Rev. E 60, 6580 (1999).

[8] S. R. Massanes and C. J. Perez Vicente, Phys. Rev. E 59, 4490 (1999).

[9] B. Lindner and L. Schimansky-Geier, Phys. Rev. E 60, 7270 (1999).

[10] J. R. Pradines, G. V. Osipov, and J. J. Collins, Phys. Rev. E 60, 6407 (1999).

[11] V. A. Makarov, V. I. Nekorkin, and M. G. Velarde, Phys. Rev. Lett. 86, 3431 (2001).

[12] B. Lindner and L. Schimansky-Geier, Phys. Rev. E 61, 6103 (2000).

[13] S. K. Han, T. G. Yim, D. E. Postnov, and O. V. Sosnovtseva, Phys. Rev. Lett. 83, 1771 (1999).

[14] Y. Horikawa, Phys. Rev. E 64, 031905 (2001).

[15] Y. Shinohara, T. Kanamaru, H. Suzuki, T. Horita, and K. Aihara, Phys. Rev. E 65, 051906 (2002).

[16] E. I. Volkov, M. N. Stolyarov, A. A. Zaikin, and J. Kurths, Phys. Rev. E 67, 066202 (2003).

[17] C. Palenzuela, R. Toral, C. R. Mirasso, O. Calvo, and J. D. Gunton, Europhys. Lett. 56, 347 (2001).

[18] Z. Liu and Y.-C. Lai, Phys. Rev. Lett. 86, 4737 (2001).

[19] Y.-C. Lai and Z. Liu, Phys. Rev. E 64, 066202 (2001).

[20] A. Neiman, L. Schimansky-Geier, A. Cornell-Bell, and F. Moss, Phys. Rev. Lett. 83, 4896 (1999).

[21] C. Zhou, J. Kurths, and B. Hu, Phys. Rev. Lett. 87, 098101 (2001).

[22] C. Masoller, Physica D 168, 171 (2002).

[23] L. I and J.-M. Liu, Phys. Rev. Lett. 74, 3161 (1995).

[24] D. E. Postnov, S. K. Han, T. G. Yim, and O. V. Sosnovtseva, Phys. Rev. E 59, R3791 (1999).

[25] G. Giacomelli, M. Giudici, S. Balle, and J. R. Tredicce, Phys. Rev. Lett. 84, 3298 (2000).

[26] A. G. Balanov, N. B. Janson, D. E. Postnov, and P. V. E. McClintock, Phys. Rev. E 65, 041105 (2002).

[27] K. Miyakawa and H. Isikawa, Phys. Rev. E 66, 046204 (2002).

[28] I. Z. Kiss, J. L. Hudson, G. J. Escalera Santos, and P. Parmananda, Phys. Rev. E 67, 035201(R) (2003).

[29] C. S. Zhou, J. Kurths, E. Allaria, S. Boccaletti, R. Meucci, and F. T. Arecchi, Phys. Rev. E 67, 066220 (2003).
[30] B. Lindner, J. García-Ojalvo, A. Neiman, and L. SchimanskyGeier, Phys. Rep. 392, 321 (2004).

[31] R. C. Hilborn and R. J. Erwin, Phys. Lett. A 322, 19 (2004).

[32] N. F. Rulkov, Phys. Rev. Lett. 86, 183 (2001).

[33] C. Morris and H. Lecar, Biophys. J. 35, 193 (1981).

[34] R. C. Hilborn (unpublished).

[35] J. G. Nicholls, A. R. Martin, B. G. Wallace, and P. A. Fuchs, From Neuron to Brain (Sinauer Associates, Sunderland, MA, 2001).

[36] N. F. Rulkov, Phys. Rev. E 65, 041922 (2002).

[37] G. de Vries, Phys. Rev. E 64, 051914 (2001).

[38] R. C. Hilborn, Am. J. Phys. 72, 528 (2004).

[39] A. Alonso and R. R. Llinás, Nature (London) 342, 175 (1989).

[40] A. Alonso and R. Klink, J. Neurophysiol. 70, 128 (1993).

[41] B. Hu and C. Zhou, Phys. Rev. E 61, R1001 (2000).

[42] P. Dayan and L. F. Abbott, Theoretical Neuroscience: Computational and Mathematical Modeling of Neural Systems (MIT Press, Cambridge, 2001).

[43] C. W. Gardiner, Handbook of Stochastic Methods (Springer, Berlin, 1985).

[44] H. Risken, The Fokker-Planck Equation (Springer, Berlin, 1989).

[45] W. Paul and J. Baschnagel, Stochastic Processes (Springer, Berlin, 1999).

[46] N. G. van Kampen, Stochastic Processes in Physics and Chemistry (Elsevier, Amsterdam, 1992).

[47] A. M. Lacasta, F. Sagues, and J. M. Sancho, Phys. Rev. E 66, 045105(R) (2002).

[48] H. Haken, Synergetics, An Introduction (Springer-Verlag, Berlin, 1983).

[49] S. H. Strogatz, Nonlinear Dynamics and Chaos (AddisonWesley, Reading, MA, 1994).

[50] W. H. Press, S. A. Teukolsky, W. T. Vetterling, and B. P. Flannery, Numerical Recipes in $C++$ : The Art of Scientific Computing (Cambridge University Press, Cambridge, England, 2002).

[51] R. C. Hilborn, Chaos and Nonlinear Dynamics (Oxford University Press, Oxford, 2000).

[52] D. Gillespie, Markov Processes (Academic Press, Boston, 1992).

[53] D. S. Lemons, An Introduction to Stochastic Processes in Physics (Johns Hopkins University Press, Baltimore, 2002).

[54] R. FitzHugh, Biophys. J. 1, 445 (1961).

[55] J. Nagumo, S. Arimoto, and S. Yoshizawa, Proc. IRE 50, 2061 (1962).

[56] W. C. Stacey and D. M. Durand, J. Neurophysiol. 86, 1104 (2001).

[57] J.-W. Shuai and P. Jung, Biophys. J. 83, 87 (2002).

[58] S. C. Tovey, et al., J. Cell. Sci. 114, 3979 (2001). 